FOCUS: MALDI-TOF AND BIOLOGICAL MS: EDITORIAL

\title{
Focus in Honor of Marvin L. Vestal, Recipient of the 2010 Award for a Distinguished Contribution in Mass Spectrometry
}

$\mathrm{T}$

he 2010 Distinguished Contribution in Mass Spectrometry (MS) Award was presented to Dr. Marvin L. Vestal (Figure 1) for his significant contributions to the theoretical understanding and development of MALDI-TOF and TOFTOF mass spectrometers, enabling these approaches as practical methods for biological applications. Even though the award was specifically focused on MALDI-TOF and TOF-TOF, Marvin has pioneered many important developments in practical MS with his extensive background in engineering and deep understanding of theory.

Marvin was born and raised in Pendleton, IN, and graduated from Purdue University with B.S. and M.S. degrees in Engineering Sciences. During his undergraduate years he took a break to join the U.S. Army Signal Corps, where he learned to copy Morse code at 30 words per minute and vastly improved his typing skills. Marvin started part-time at Johnston Laboratories in Lafayette, IN, where he worked with Henry Rosenstock and Merrill Wallenstein who, as students of Austin Wahrhaftig and Henry Eyring, had developed the quasiequilibrium theory (QET) of MS. Marvin collaborated with Rosenstock in developing the Coincidence Time-of-Flight mass spectrometer and invented a secondary electron multiplier that improved the performance of this instrument. He also worked with Wahrhaftig to correct a major problem with the original QET and applied the theory to calculations on fragmentation of "large" molecular ions such as propane. This work was supported by the Air Force Aeronautical Research Laboratory and was monitored by Lt. Jean Futrell.

Marvin moved to Baltimore along with Johnston Laboratories in 1960 and attended graduate school at Johns Hopkins in Physics, but dropped out when it was clear that none of the faculty were interested in any system larger than an atom. In 1967, Scientific Research Instrument Corporation (SRIC) was founded by Bill Johnston, Gordon Fergusson, Bob Jones, and Marvin. At that time, the chemical ionization (CI) method had just been introduced by Frank Field and Burnaby Munson. SRIC licensed the CI technique from Humble Oil (now Exxon), and Marvin built the first commercial CI source at the request of Henry M. (Hank) Fales at the National Institutes of Health

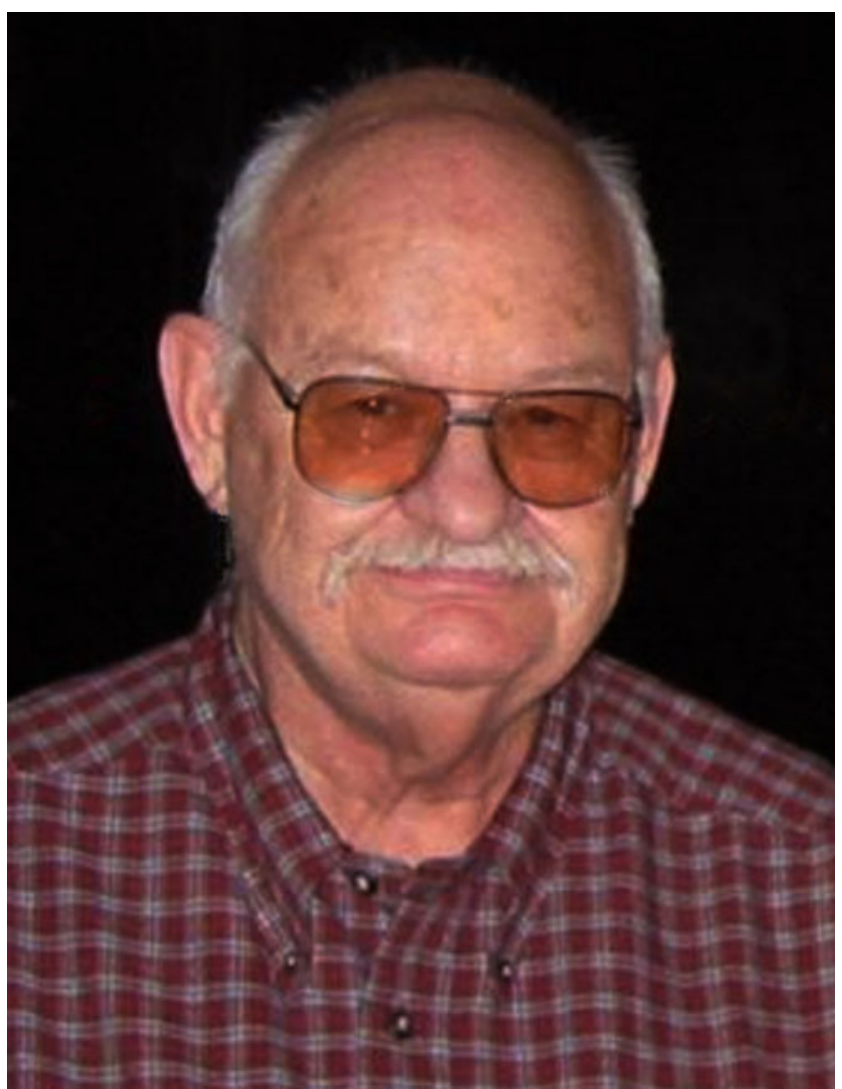

Figure 1. Marvin L. Vestal

(NIH), developing a friendship that lasted until Hank's recent death. Marvin hired Alfred Yergey (currently at NIH) to work at SRIC as his replacement when he left to pursue a Ph.D. in 1970.

Marvin became a student of both Jean Futrell and Austin Wahrhaftig in the Chemical Physics program at University of Utah. There have been few graduate students who are independently established in a field and write proposals to obtain their own research grant. Marvin built a crossed-beam apparatus for his Ph.D. thesis, and the first tandem quadrupole mass spectrometer to study photodissociation mechanisms. 
While finishing his thesis, Marvin obtained NIH RO1 funding, with a substantial boost from Jim McCloskey, and began to build an LC-MS instrument that was later moved to the University of Houston (Figure 2). McCloskey moved from Houston to Utah about the time that Marvin completed the Ph.D., and he and Marvin established a long collaboration. Marvin appears to have enjoyed greatly his graduate-school days, formulating new theories of ion-molecule reactions, building new instruments, expanding his scientific circle of friends and collaborators, and smoking cigars (Figure 3).

With a successful RO1 grant, Marvin had little difficulty in securing a position at the University of Houston as an Associate Professor of Chemistry in 1976. Calvin Blakley, who joined Futrell's group in 1972 as a postdoctoral fellow, was persuaded to accompany Marvin and became a member of "Vestal's Virgins," a title conferred by Hank Fales (Figure 4). Calvin remained a long-time colleague of Marvin, and they developed various instruments together until Marvin moved his operation to Framingham, MA.

Marvin's work in Houston was focused on the most important interfacing problem for pharmaceutical and biomedical analysis: that of liquid chromatography to MS. Gas chromatography-mass spectrometry (GC-MS) interfacing had been implemented for about a decade, and commercial instruments had been in operation in many laboratories. However, most biomolecules of interest were not amenable to GC-MS since the technique requires volatilization of molecules before ionization. Coupling LC with MS was a much greater challenge because the mass transfer rate by LC was orders of magnitude higher than could be accommodated by the vacuum systems of most mass spectrometers. In addition, new soft ionization techniques that were compatible with LC-MS were still needed for analyses of nonvolatile and polar biomolecules. Marvin and co-workers developed the Thermospray technique originally as a vaporization method to introduce the samples eluting from an LC into a CI mass spectrometer. However, they soon found that when the mobile phase contains a significant concentration of electrolytes, the Thermospray itself serves as an ionization method, producing abundant molecular ion species with few

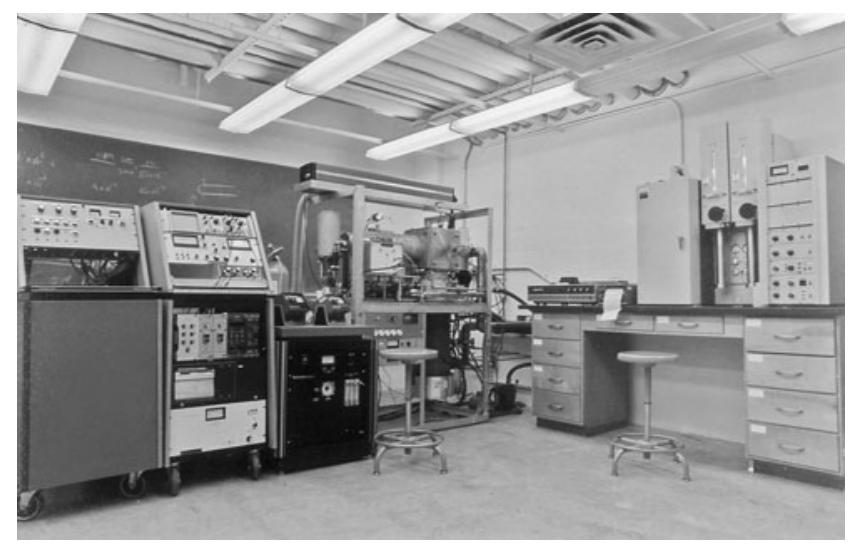

Figure 2. The first LC-MS instrument Marvin began to build in Utah and later moved to University of Houston

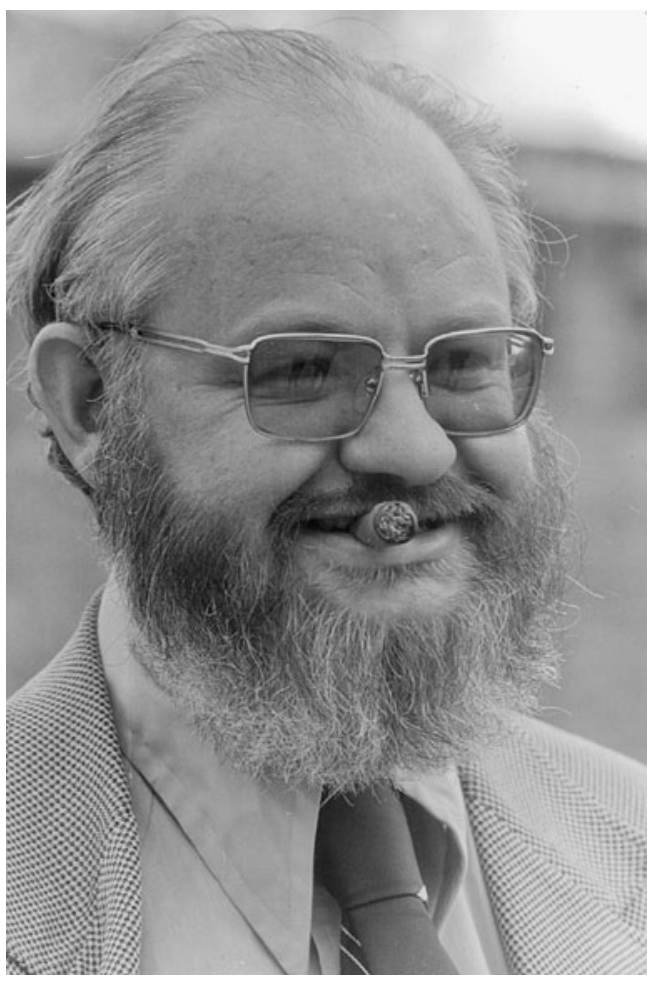

Figure 3. Marvin at graduation in 1975

fragments and low and predictable background. Marvin with his co-workers published seminal papers on this development in Anal. Chem., JACS, and Clin. Chem. (1980). They not only provided a critical innovation in analytical chemistry but also discovered a fundamentally new ionization method. Marvin relentlessly improved the performance of Thermospray through many reiterations of heated-capillary vaporizers and ion-source configurations. In fact, the ever-evolving Thermospray LC-MS instrument in Marvin's laboratory never stayed in one configuration for more than a few weeks in the early 1980s. In between those modifications, Marvin and colleagues successfully demonstrated the power of Thermospray LC-MS in cutting-edge on-line

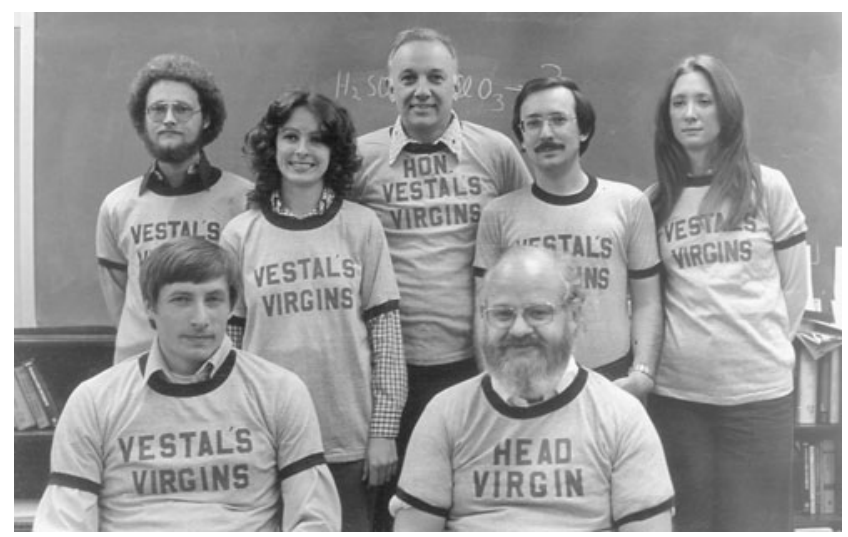

Figure 4. Vestal's Virgins. From left to right; Dwayne Hardin, Calvin Blakley, Mary Jane McAdams, Henry Fales, Marvin Vestal, Joe Hiller, and Julie Robeson 
peptide sequencing by LC-MS (published in Anal. Chem., JACS, Science, 1984).

Since the promise of convenient analysis of biological compounds, drugs, and drug metabolites directly from clinical specimen was just too great to overlook, Marvin founded his own company, Vestec, to produce commercial LC-MS instruments based on the Thermospray interface. Soon, this technique became the first practical LC-MS interface, enabling direct analysis of biological samples and drawing enormous attention from the life-sciences community. Indeed, the commercial Thermospray instruments helped LC-MS to be recognized for the first time by non-mass spectrometrists as one of the most powerful techniques. In addition, Marvin's studies of ion evaporation/desorption mechanisms involved in Thermospray ionization provided a fundamental understanding about common traits in spray ionization (Mass Spectrom. Rev., 1983). Furthermore, his successful applications of Thermospray to biologically important and relevant nucleosides and peptides heightened the interest of the biomedical communities and prompted further development of the interface. Soon thereafter, other soft ionization techniques, such as electrospray and matrixassisted laser desorption ionization, were introduced, and they later were recognized by Nobel-Prize awards to the inventors. Electrospray ionization (ESI) invented by John Fenn, in particular, provided a far more stable LC-MS interface; a stable interface was critically important to the broad user base created by the commercial Thermospray LC-MS instruments. Although Thermospray "passed the torch" to ESI, it may not be an overstatement to say that Marvin set the stage for practical LC-MS in life sciences and paved the way for ESI.

Following the invention of MALDI by Karas and Hillenkamp, Marvin sensed the great potential of MALDI-TOF for commercial instruments for the life-sciences community. TOF analyzers, the natural match for MALDI, are perhaps the simplest of all mass analyzers and relatively straightforward to use. From then on, Marvin's focus became developing MALDI instruments with TOF analyzers. He and his colleagues at Vestec built the first commercial MALDI-TOF instrument in 1990, and Vestec's acquisition by PerSeptive Biosystems (later merged to Applied Biosystems Incorporation, $\mathrm{ABI}$ ) expanded its commercial viability.

However, a remaining problem was that MALDI-TOF had low performance; mass resolving power was marginal, accuracy of mass measurements was coarse, and the lack of MS-MS functionality was a substantial limitation. Marvin systematically addressed all of these issues over the following decade. In 1994, Lennon and Brown reported significant improvements in the resolving power of MALDITOF by applying an extraction pulse with precise delay after the ionizing laser pulse. It took barely a year for Marvin to connect the dots from previously published velocity distributions of MALDI-generated ions to focusing requirements in linear and reflector time-of-flight analyzers. Marvin's most significant accomplishment probably was the development of a comprehensive theoretical model of the various components of a TOF analyzer with a view toward optimizing performance of a complete system for particular applications (Rapid Commun. Mass Spectrom., 1995; J. Am. Soc. Mass Spectrom., 1998). The first application of this theoretical approach was the design of a family of MALDITOF instruments employing delayed extraction. The resulting Voyager series of instruments provided the scientific community access to MALDI-TOF with mass resolving power exceeding 10,000 and mass accuracy with sub-10ppm mass errors. More than a half of the MALDI-TOF instruments in use today are based on Marvin's designs.

The last frontier for MALDI-TOF was the lack of sensitive MS-MS capability. The metastable decay in MALDI-TOF instruments characterized by Kaufmann and Spengler could not achieve the analytical accuracy of MSMS measurements by triple-quadrupole, ion trap, or hybrid TOF instruments. By refining his theory and extending it to the design of tandem TOF, Marvin successfully constructed MALDI-TOF-TOF instruments, which were introduced commercially by Applied Biosystems as the 4700 Proteome Analyzer, and later as the 4800 TOF-TOF; these instruments are used today in most proteomics centers. Both MALDITOF MS and MS-MS systems have had an enormous impact on many important areas of research that include proteomics, glycomics, cell signaling, structural biology, organelle imaging, and polymer science, to name just a few.

There are few scientists and innovators who have had significant impact on their field both through research and commercial technology development. Perhaps no one has had a more profound dual impact on modern mass spectrometry than Marvin during his career spanning four decades. The American Chemical Society (ACS) recognized his significant contribution with the Frank H. Field and Joe L. Franklin Award for Outstanding Achievement in Mass Spectrometry in 2005. In 2010, Marvin's development of comprehensive theoretical models for the various components of time-of-flight analyzers and application of this theory to the design and optimization of MALDI-TOF and TOF-TOF mass spectrometers for biological applications was recognized by the ASMS with the Award for a Distinguished Contribution in Mass Spectrometry. As the ASMS has been the major society for his scientific activities for more than 40 years, this award has very special meaning for Marvin. He is truly one of the leading lights in mass spectrometry, and he never seems to tire of translating theory into practical commercial instruments. After the "retirement" from ABI in 2004, Marvin started a new company, Virgin Instruments, with his wife Christina (Figure 5), and he still continues to develop MALDI-TOF-TOF instruments, taking advantage of recent advances in related technology and his theoretical predictions (J. Mass Spectrom. 2008).

Organization of the Focus: This JASMS Special Focus begins with a Critical Insight article "The Future of Biological Mass Spectrometry" by the Distinguished Contribution awardee; in this paper, Marvin argues that recent developments in time-of-flight technology, coupled with 


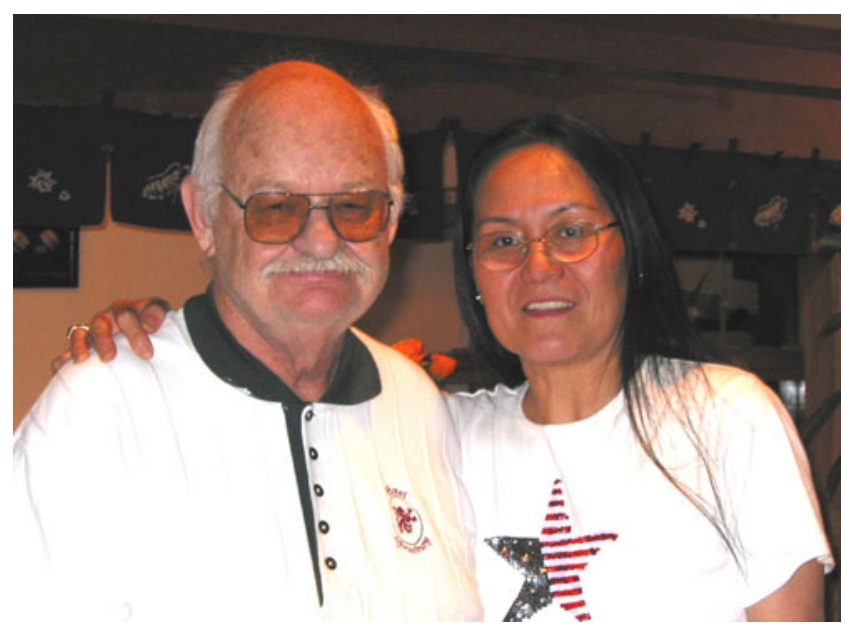

Figure 5. Marvin and Chris Vestal

MALDI techniques, can provide a powerful approach to addressing the current challenges in functional proteomics and metabolomics. The collection of subsequent articles reflects the significant impact of Marvin's theoretical and instrumental innovations in various research fields; the papers were contributed by colleagues and collaborators who have benefitted from Marvin's pioneering developments. Randy Nelson describes his work with Marvin to construct the "inaugural instrument in the world's first commercial line of MALDI-TOF mass spectrometers;" he and Chad Borges then provide an overview of mass spectrometric immunoassay (MSIA) and its intrinsic link to MALDI-TOF. Al Yergey and co-workers demonstrate the application of the quality threshold clustering algorithm to produce reliable MALDI spectra, Michael Karas and Thorsten Jaskolla discuss evidence for a unified MALDI analyte protonation mechanism, and Peter Roepstorff and colleagues describe combined fractional diagonal chromatography and off-line nano-LC-MALDI to identify nitrated peptides. In the following paper, Catherine Costello and Liang Han apply electron transfer dissociation to the structure identification of carbohydrates for the first time. The final two papers combine MALDI-TOF with imaging mass spectrometry. Robert Murphy and collaborators characterized the spatial distribution of lipid molecular species in rat brains for ischemia and traumatic brain injury models. Richard Caprioli and Jeffrey Spraggins utilized a prototype MALDI-TOF built by Marvin and his team at Virgin Instruments to demonstrate high-speed ion image acquis-

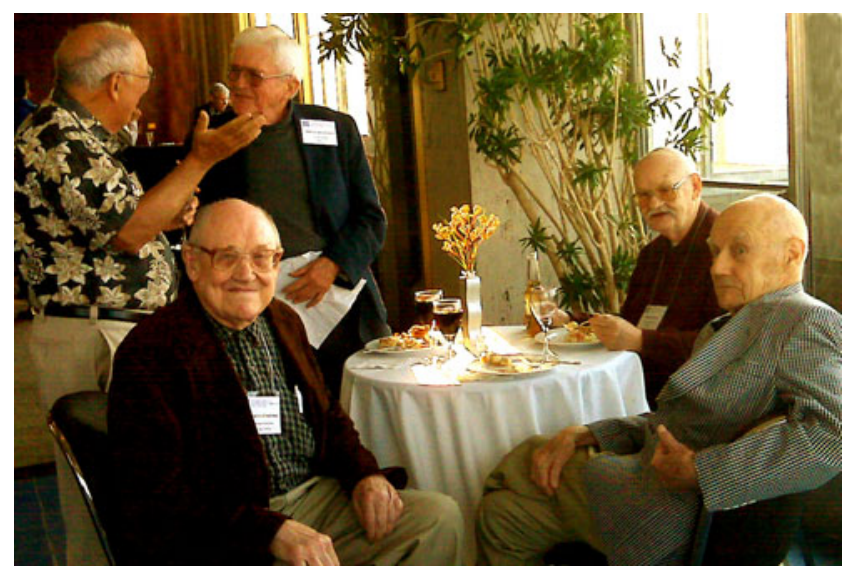

Figure 6. Good old friends at Marvin's birthday celebration in 2009. From left to right: Mike Story, Ken Standing, Fred McLafferty, Marvin, and John Fenn

ition; this development is a prelude to the next generation of MALDI imaging mass spectrometry instruments that must address applications of increased spatial resolution and sample size.

Since Marvin's tenure at the university was brief, he produced fewer students than most academic researchers. Nevertheless, he made numerous scientific friends and colleagues along his scientific journey who cherish and respect him and his contributions (Figure 6); this JASMS Associate Editor is pleased to be numbered among his longtime friends. To his students and colleagues, most of whose careers are indebted to his reputation, he was a generous mentor and warm friend. As one of his few students, this Guest Editor feels immensely fortunate to have studied under him and known him personally. It was truly an honor and privilege to prepare this focus editorial for Dr. Marvin L. Vestal, who will be my mentor forever. Congratulations, Marvin!

\section{Hee-Yong Kim}

Guest Editor, NIAAA, NIH

Bethesda, Maryland

e-mail: hykim@nih.gov

Veronica M. Bierbaum

Associate Editor, JASMS

University of Colorado

Boulder, Colorado

e-mail: Veronica.Bierbaum@Colorado.EDU 